Available online at GSC Online Press Directory

GSC Biological and Pharmaceutical Sciences

e-ISSN: 2581-3250, CODEN (USA): GBPSC2

Journal homepage: https://www.gsconlinepress.com/journals/gscbps

(REVIEW ARTICLE)

\title{
Antiplatelet drugs overview
}

\author{
Latif Sarah Younis *, Issa Mustafa Mohammed, Hassan Tabark Najah and Ali Murtadha Haider \\ College of Pharmacy, Al-Nahrain University, Iraq.
}

Publication history: Received on 07 January 2020; revised on 15 January 2020; accepted on 19 January 2020

Article DOI: https://doi.org/10.30574/gscbps.2020.10.1.0003

\begin{abstract}
Platelets are essential factor in the pathophysiology of atherothrombosis, abnormal platelet function guiding to many cardiovascular complications such as myocardial infarction and ischemic stroke. There are numerous antiplatelet drugs approved for use in the clinical management and several under examination. Antiplatelet drugs with the high potency are mostly associated with the intensifying incidence of bleeding. Aspirin and clopidogrel (alone or dual) are the mostly used drugs with the best favorable risk-benefit profiles of drugs available. While cangrelor and ticagrelor may produce further benefits but need more studies .Other drugs such as prasugrel, dipyridamole and cilostazol are not widely used.
\end{abstract}

Keywords: Aspirin; Thienopyridines; Coronary heart disease

\section{Introduction}

Platelets are vital parts of normal hemostasis and key components in atherothrombosis .Therefore, the diseases that associated with thrombus formation such as arteriosclerosis are treated mainly by antiplatelets drugs [1].

Platelets are non-nucleated cells that formed from megakaryocyte and have a maximum circulating life span of 7-10 days. Adjustment of platelet production is mediated by thrombopoietin, which is produced by liver, bone marrow and kidney, binding to high-affinity receptors on platelets and megakaryocytes [2].

Platelets provide a circulating source of chemokines, cytokines, and growth factors, which are preformed and packaged in storage granules. Although platelet adhesion, activation, and aggregation regarded as a normal repairing response to the sudden fissuring or rupture of an atherosclerotic plaque, but uncontrolled progression of such process can cause intraluminal thrombus formation, vascular occlusion, and subsequent ischemia or infarction [3].

As noted in figure 1, Thrombin binds to protease activated receptor1(PAR-1), which leads to shape change, phospholipase C (PLC) activation, thromboxane A2 (TXA2) generation, and activation of the glycoprotein (GP) IIb/IIIa receptor, resulting in sustained platelet aggregation. Cyclooxygenase (COX)-1 catalyzes the production of TXA2, a potent platelet aggregator, generated by platelets activated by thrombin and other agonists [4].

Adenosine 5'-diphosphate (ADP) binds to its 7-transmembrane domain receptors, P2Y1 and P2Y12, to activate platelets.

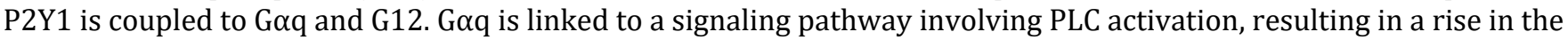
intracellular calcium concentration ion $\left[\mathrm{Ca}^{+2}\right]$ and protein kinase C (PKC) activation, leading to GP IIb/IIIa activation and transient platelet aggregation [4].

\footnotetext{
${ }^{*}$ Corresponding author

E-mail address: younissarah96@gmail.com
} 
G12 mediates platelet shape change. P2Y12 is linked to Goi-coupled signaling cascades associated with adenylcyclase (Ac) down-regulation and decreased cyclic-3', 5'-monophosphate (cAMP) production, which mediates GP IIb/IIIa receptor activation, leading to sustained platelet aggregation [4].

Although activated platelets are incapable of de novo protein synthesis, they can translate constitutive mRNA into protein over the course of several hours. Thus, platelets may play a role in inflammation, angiogenesis, and wound healing, and antiplatelet therapies may have an impact on these processes by blocking platelet-derived protein signals for inflammatory or proliferative responses [5].

In addition to cardiac troponin I, Myeloperoxidase is another test used for the inspection of the existence of inflammatory reaction and oxidation that escorted thrombus formation and aggravated acute atherosclerosis symptoms [6].

Antiplatelet drugs are indicated for the management of thrombotic diseases that included stroke, acute myocardial infarction (AMI), acute coronary syndrome (ACS), angina, percutaneous coronary intervention (PCI), cardiac surgery, primary and secondary cardiovascular disease prevention, peripheral vascular disease, and thrombotic disorders such as atrial fibrillation.

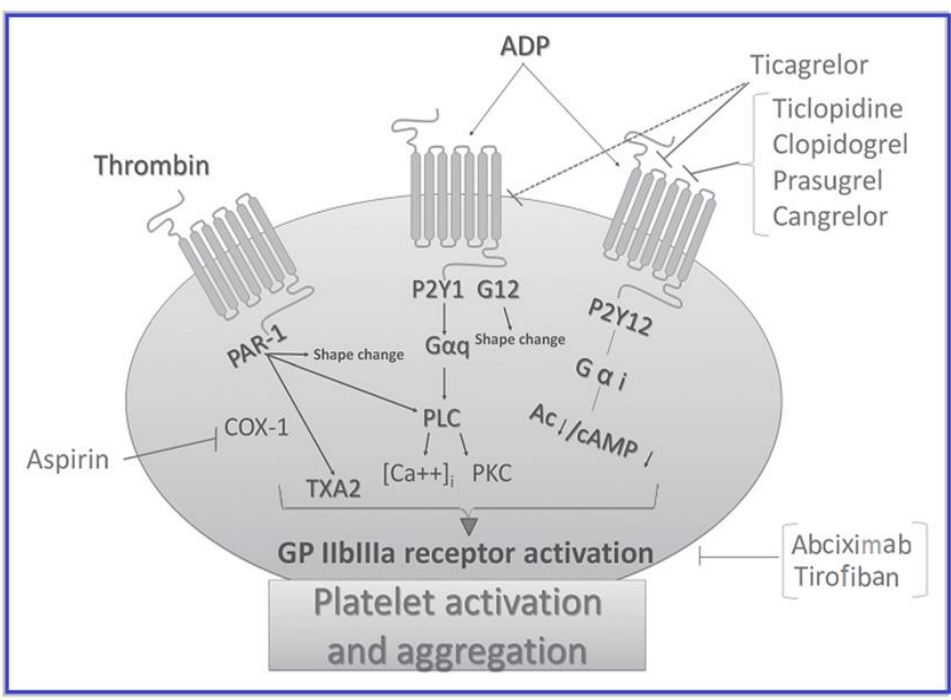

Figure 1 Platelet activation pathways and antiplatelet drugs targeting [4]

\section{Classes of antiplatelet drugs}

\subsection{Platelet cyclooxygenase (COX-1) inhibitor}

\subsubsection{Aspirin}

Aspirin is the backbone treatment for all state of atherothrombosis. The effectiveness of aspirin on platelet came from its ability to irreversibly inhibit platelet cyclooxygenase (COX-1), by acetylating a serine located near the active site of the enzyme, that is responsible for the conversion of arachidonic acid to prostaglandin (PGH2) and thereafter production of thromboxane A2 (TxA2) and prostocycline (PGI 2) [3].

Human platelets and vascular endothelial cells form PGH 2 to produce primarily TXA 2 and PGI 2, respectively. TXA 2 induces platelet aggregation and vasoconstriction, whereas PGI 2 inhibits platelet aggregation and induces vasodilation .Fulfilled inhibition of platelet COX-1 and then TXA 2 can occur with low daily doses of aspirin (75-150 mg). Conversely, the endothelium COX-2 generates PGI2 and is less sensitive to aspirin inhibition. As a result, low-dose aspirin has limited effects on PGI2-dependent vascular functions including arterial blood pressure regulation [7].

Aspirin is rapidly absorbed after oral administration in the stomach and upper intestine.it is achieved plasma peak levels on 30 - $40 \mathrm{~min}$. and it is inhibit platelet function within $1 \mathrm{hr}$. while enteric-coated aspirin form take more time to reach plasma peak levels. The lower bioavailability of some enteric-coated preparations and their poor absorption from small intestine may result in inadequate platelet inhibition when these preparations are used at low doses [8]. 
Aspirin irreversibly inactivates platelet COX-1, therefore in spite of the rapid clearance of aspirin from the circulation, the platelet-inhibitory effects last for the life span of the platelet (10 days).Additionally, aspirin acetylates megakaryocyte COX-1, thereby inhibiting thromboxane production in newly released platelets [9].

The major adverse effect of aspirin intake is enhanced risk of bleeding tendency especially by gastrointestinal tract although this risk may be amended by the use of gastro protective drugs such as proton pump inhibitors (PPIs) [10]. The concomitant intake of nonselective reversible COX-1 inhibitors such as ibuprofen or naproxen with aspirin can interfere with the antiplatelet effect of low-dose aspirin and performing aspirin less effective when used for cardioprotection or stroke prevention. Interestingly, a competition exist between the nonselective COX inhibitors and aspirin for the common binding site within the COX-1 channel, which may prevent aspirin from acetylating the serine residue and lead to aspirin resistance [11].

The incapability of aspirin to inhibit COX-1-dependent TXA2 production is called aspirin resistance, and it is about 12\%. High On-Treatment Platelet Reactivity (HPP) or resistance to antithrombotic drug is consider as an adverse thrombotic events or treatment failure which is associated with increase the risk of MI, stroke, or death [12].

Many factors effect on the platelet reactivity such as age, female sex, diabetes, concomitant therapies (particularly NSAIDs, e.g, ibuprofen). Conditions that associated with an inflammatory response such as unstable angina, acute myocardial infarction (AMI), diabetes, and cardiac surgery are associated with HPR in aspirin-treated patients [13].

\subsection{Platelet P2Y12 -ADP receptor irreversible inhibitor (Thienopyridines)}

Thienopyridines class involved three generations, ticlopidine, clopidogrel, and prasugrel. All of them are prodrugs that must converted by metabolic activation through the hepatic CYP450 system to their active metabolites that selectively inhibit the platelet P2Y12 -ADP receptor [14].

\subsubsection{Ticlopidine}

The first generation thienopyridines,Ticlopidine, has limited use due to its bone marrow toxicity, neutropenia, aplastic anemia, and thrombotic thrombocytopenic purpura [15].

\subsubsection{Clopidogrel}

Clopidogrel, a second generation thienopyridine, is a prodrug that irreversibly binds to the P 2 Y 12-ADP platelet receptor by forming disulfide bridges after a two-stage of activation by cytochrome P450 (CYP) liver isoenzymes. Conspicuously, clopidogrel is more safer than ticlopidine, and is more effective than aspirin for prevention of secondary vascular events .As clopidogrel and aspirin act on discrete and complementary pathways of platelet inhibition, dual therapy is evaluated in high-risk clinical conditions [16].The use of clopidogrel is certified for the reduction of atherosclerotic events in diabetic patients and in patients with recent stroke, recent MI,or established peripheral arterial disease $[17,18]$.

After oral administration of clopidogrel, the drug is variable absorbed. 108 and the majority of absorbed clopidogrel $(85 \%)$ is extensively hydrolyzed by esterases to the inactive carboxylic acid metabolite. In the liver, clopidogrel is metabolized in a 2-step process by CYP3A4/3A5 and CYP2B6 / 1A2/2C9/2C19 to a very short-lived active metabolite, which is responsible for its effect on platelet aggregation [19].

Peak concentrations of the parent drug, its active metabolite and the carboxylic acid metabolite occur within 1- $2 \mathrm{hr}$ .The drug and its metabolite are extensively bound to serum proteins. Elimination is by the feces (50\%) and urine (50\%). Inhibition of platelet aggregation reaches a steady state $50-60 \%$ inhibition after $4-7$ days of daily administration of $75 \mathrm{mg}[20]$.

There are interpatient variability's in response to clopidogrel and thereafter in antiplatelet activity ,the most important one is the genotype variability of liver cytochrome enzyme e.g.CYP2C19 genotypes are associated with diminished platelet response to clopidogrel but this may be overcome by monitoring and adjusting the dose based on the platelet reactivity [21].

Loading dose of $300 \mathrm{mg}$ of clopidogrel results in more-rapid platelet inhibition than is achieved with the $75 \mathrm{mg}$ maintenance dose. Moreover, inhibition of ADP-induced platelet aggregation was also significantly greater with a 600mg loading dose of clopidogrel compared with a 300-mg loading dose [22]. 
The major side effect of clopidogrel administration is the increased risk of bleeding. Compared with aspirin, there were fewer GI symptoms but an increased incidence of diarrhea and rash. A rare but significant complication of clopidogrel is the development of thrombotic thrombocytopenic purpura [23].

Because of the requirement for metabolism of clopidogrel by CYP3A4/3A5 to generate the active metabolite, many drugs that metabolize by CYP3A or CYP2C19, e.g. statins drug or proton pumps inhibitors drugs respectively can interfere with the metabolism of clopidogrel and thereafter with its clinical effect. Concurrent used of atorvastatin with clopidogrel may provoke hepatic injury cholestasis type resulting from abnormal bile flow caused by either drugs or their metabolites [24]. Additionally, atorvastatin has been found to amend the functions of endothelium by its effect on peroxisome -proliferator-activated receptors (PPAR- $\alpha$ ) pathway [25].

Erythromycin, calcium antagonists, macrolide antibiotics, or ketoconazole,CYP3A inhibitors, can produce lessening in antiplatelet activity of clopidogrel .While Rifampin, a CYP3A inducer, can reveal enhancing antiplatelet activity of clopidogrel [26].

Many studies found that there are variability in patient's response to clopidogrel and may be associated with adverse thrombotic events especially in those with high on-treatment platelet reactivity. Particularly, Several factors can associate with increased incidence of high platelet reactivity in patients with clopidogrel such as diabetes, dyslipidemia, concomitant therapies that effect on metabolism of clopidogrel and related to insufficiency of active metabolite such as (lipophilic statins, e.g, simvastatin and atorvastatin; proton pump inhibitors, eg, omeprazole; and calcium channel blockers), and genetic polymorphisms of CYP450 isoenzymes (2C19, 1A2, 2B6, 2C9, 3A4) that involved in the production of clopidogrels' active metabolite [27].

\subsubsection{Prasugrel}

Prasugrel, a prodrug of the thienopyridine family, after a rapid one-step conversion by CYP3A4 and to a lesser extent CYP2B6 into a highly bioavailable metabolite, causes an irreversible block of the P2Y12 ADP receptor prevent platelet activation. Because of a distinct chemical structure, the conversion to its active metabolite is less dependent on specific cytochrome P450 enzymes than that of other thienopyridines [28].

In patients with stable coronary artery disease, prasugrel produced a faster and more effective inhibition of platelet function than clopidogrel. The incidence of poor platelet aggregation response after prasugrel $60 \mathrm{mg}$ administration was lower than for clopidogrel $300 \mathrm{mg}$ [29].

Prasugrel is rapidly absorbed after oral ingestion, unaffected by food, and is rapidly converted to its active metabolite, which reaches peak concentrations within $30 \mathrm{~min}$ of dosing. The active metabolite has a half-life of $4 \mathrm{~h}$, and renal excretion is the major route for elimination of the metabolites. Prasugrel is gave as $60 \mathrm{mg}$ loading and $10 \mathrm{mg}$ maintenance doses [30].

The major adverse effect of prasugrel is bleeding. Prasugrel is a more potent inhibitor of platelet function than clopidogrel and therefore it associated with a significantly increased incidence of major adverse bleeding events and should be avoided in patients with known cerebrovascular disease [31]. There were a little probability of interactions with other drugs metabolized by cytochrome P450 system (CYP3A4/CYP2B6) as reported in many studies [32].

Compared with clopidogrel, use of prasugrel cause few nonresponders and better clinical response in diabetic patients. Poor response to clopidogrel was attributed to reductions in the amount of measured active metabolite available to interact with platelets as opposed to alterations in the platelet P2Y12 receptor [33].

\subsection{ADP- receptor antagonists}

\subsubsection{Cangrelor}

Cangrelor, an adenosine triphosphate (ATP) analog, is a reversible inhibitor of the platelet P2Y12- ADP receptor. It achieves greater inhibition of platelet aggregation than that obtained by clopidogrel. But other study found that the measurement of inhibition of platelet aggregation did not exhibit any variation between cangrelor and clopidogrel [34, $35]$.

Cangrelor present as IV-form, has a rapid onset of action (steady state at $30 \mathrm{~min}$.) and elimination half-time of $9 \mathrm{~min}$. Moreover, platelet activity rapidly return to its normal state within 60 minutes after interruption of drug. Cangrelor 
metabolized by sequential dephosphorylation in plasma therefore it can be used in patients with renal or hepatic function abnormalities [36].

The most common adverse effects of cangrelor treatment include, bleeding, transient increases in liver enzymes, and dyspnea [37]. Cangrelor can interact with the platelet inhibitory activity of clopidogrel at active site on platelet when given concurrently. Therefore, clopidogrel can be gave consecutively after cangrelor. Hence, cangrelor might consider as P2Y12 inhibitor bridge therapy in the perioperative and operative period [38].

\subsection{Platelet P2Y12 -ADP receptor reversible inhibitor}

\subsubsection{Ticagrelor}

Ticagrelor, an orally active cyclopentyl-triazolopyrimidine, binds to the P 2 Y 12 receptor, other than those recognized by ADP, in a reversible manner and almost completely inhibits ADP-induced platelet aggregation. It has a faster onset and offset of platelet inhibition than clopidogrel [39].

After oral administration, ticagrelor is rapidly absorbed and does not require hepatic biotransformation to be pharmacologically active. It is peak effect on platelet inhibition was $2-4$ hours. The terminal half-life was approximately 7 hours. of addition, ticagrelor is also metabolized to an equipotent, active metabolite by CYP $3 \mathrm{~A} 4$ enzymes thereafter both ticagrelor and its active metabolite excreted by the intestinal route, no dose adjustment is required in kidney failure. ticagrelor is given in a loading dose of $180-270 \mathrm{mg}$ daily and in a maintanence dose of 90-mg twice daily to optimize its efficacy, safety, and tolerability [40].

The most common adverse event with ticagrelor intake is bleeding .Major, life-threatening, or fatal bleedings did not differ between those on ticagrelor and those on clopidogrel. Another adverse events is dyspnea which is reversible and required cessation of therapy when begin [41].

A reversible increase in serum uric acid and creatinine also noted. Additionally, nausea, hypotension, and asymptomatic ventricular pauses may occur because of an adenosine-mediated response. Therefore, ticagrelor treatment was precautioned in patients with hyperuricemia, bradyarrhythmias without pacemakers, and syncope (fainting) and in those at high risk of bleeding (e.g. elderly, low bodyweight, renal dysfunction) and avoided in patients with history of stroke [42].

\subsection{Phosphodiesterase inhibitor drugs}

\subsubsection{Dipyridamole}

Dipyridamole is a pyrimidopyrimidine derivative with vasodilator and antiplatelet properties. It is act by inhibition of nucleotide phosphodiesterase, an enzyme that destroys cyclic adenosine monophosphate (cAMP), causing increase in intraplatelet cyclic AMP which resulted in inhibition of platelet aggregation and blockade of adenosine uptake thereby increasing the amount of adenosine at the platelet vascular interface. In addition, dipyridamole directly stimulate the synthesis and release of prostacyclin (PGI2) from the endothelium [43].

After oral administration, dipyridamole is variably absorbed from gastrointestinal tract that lead to variable levels of its systemic bioavailability. Recently this feature has been amended with the addition of low-concentration of aspirin to dipyridamole and form a sustained release drug. Dipyridamole is greatly bound to albumin, exposed to enterohepatic recirculation, after conjugated to glucuronide it's mainly excreted by bile [44].

Use of dipyridamole is associated with an increased risk of bleeding events. The most common adverse effect of chronic administration is headache. In high doses, dipyridamole -induced vasodilation and tachycardia may produce myocardial ischemia, which may be a limiting factor for its use as an antiplatelet drug [45].

The dual therapy of dipyridamole and aspirin caused increase the risk of headache and bleeding. Moreover, As dipyridamole has vasodilator properties therefore additive vasodilation and hypotensive effects may occur when taken with other vasodilator drugs such as angiotensine converting enzyme (ACE) inhibitors inhibitors [46].

\subsubsection{Cilostazol}

Cilostazol, 2-oxoquinolone derivative, selectively inhibit intracellular phosphodiesterase type 3 enzyme that sequently lead to inhibit platelet aggregation and vasodilation. Cilostazol intake in a dose of $50 \mathrm{mg}$ bid or $100 \mathrm{mg}$ once daily was found to increase maximal and pain-free walking distance in patients with intermittent claudication [47], and averts 
stent thrombosis and restenosis [48]. In diabetic patients on standard dual antiplatelet therapy (aspirin and clopidogrel), adjunctive treatment with cilostazol enhances inhibition of platelet P 2 Y 12 signaling [49].

Orally intake cilostazol has variable level of absorption. It is abundantly bound to albumin and metabolized primarily by CYP3A $4 / 5$ to inactive metabolites that eliminated by urine.The elimination half-time for cilostazol is approximately 10 hours [50].

Cilostazol treatment especially at the first -two weeks caused gastrointestinal side effects, and headache that was the basis of drug interruption by some patients. Similarly to dipyridamole, Cilostazol caused hypotension and tachycardia as a result of vasodilation. Therefore, it is contraindicated in patients with heart failure [51].

As cilostazol metabolized by CYP3A4 and CYP2C19 therefore, it may interfere with other drugs metabolized by these enzymes such as omeprazole (CYP2C19 inhibitor) or erythromycin (CYP3A4 inhibitor) that may lead to deteriorate its biological level [52].

\subsection{GpIIb-IIIa receptor antagonists}

This class of drugs involve Abciximab (monoclonal antibody) and Tirofiban (non-peptide tyrosine derivative) both are intake as IV bolus dose and are selectively bounds to GpIIb-IIIa receptor of platelet and inhibit its binding to fibrinogen and aggregation [53].

\subsubsection{Abciximab}

Abciximab is a monoclonal antibody inhibits not only GP IIb-IIIa receptor but also GP Ib receptor for von Willebrand factor (vWF) on platelets, thereby decreasing aggregation through fibrinogen and adhesion through vWF [54].

After IV bolus administration $(0.25 \mathrm{mg} / \mathrm{kg})$, abciximab rapidly bound to GpIIb-IIIa and $80 \%$ of the GpIIb-IIIa receptors blocked and platelet aggregation diminished to $20 \%$ of baseline. Half-life of equal to about $30 \mathrm{~min}$. After $2 \mathrm{hr}$. the clinical effect of abciximab was observed and after $24 \mathrm{hr}$. the platelet activity and bleeding time regularly restore to normal [55].

It is used to decrease ischemic events of managed acute coronary syndrome and as adjunctive therapy during percutaneous coronary intervention (PCI), but trials with orally administered GP IIb-IIIa inhibitors have failed to demonstrate any benefit [56]. Thrombocytopenia is the main adverse effect that showed in $1-2 \%$ of the treated patients and with re intake of the drug the incidence of thrombocytopenia elevated [55].

\section{Conclusion}

Antiplatelet drugs are used to avoid the formation of platelet-rich arterial thrombi, while anticoagulants drugs are used to avert the formation of fibrin-rich thrombi such as left atrial appendage thrombi. The appearance of many adverse effects of Antiplatelet drugs such as bleeding, and inter individual response variability enhanced the requirement for laboratory and genetic information to pursue the therapeutic monitoring for each patients.

\section{Compliance with ethical standards}

\section{Acknowledgments}

The authors would like to impart their sincere thanks to the Dean of Pharmacy College, Al-Nahrain University in Iraq, for the strengthening to execute this Article.

\section{Disclosure of conflict of interest}

No conflicts of interests to assert.

\section{References}

[1] Bhatt DL. (2009). Role of antiplatelet therapy across the spectrum of patients with coronary artery disease. American journal of cardiology, 103, 11A-9A. 
[2] Kaushansky K. (2009).Determinants of platelet number and regulation of thrombopoiesis. HematologyAmerican Society of Hematology Education Program, (1), 147 - 152.

[3] Davì G and Patrono C. (2007). Platelet activation and atherothrombosis. New England Journal of Medicine, 357(24), 2482 - 2494.

[4] Di Minno MND, Guida A, Camera M, Colli S , Di Minno G, and Tremoli E. (2011).Overcoming limitations of current antiplatelet drugs: A concerted effort for more profitable strategies of intervention. Annals of Medicine, 43, 531544.

[5] Lindemann S, Tolley ND, Dixon DA, McIntyre TM, Prescott SM, Zimmerman GA, et al . (2001).Activated platelets mediate infl ammatory signaling by regulated interleukin 1-beta synthesis.Journal of Cell Biology, 154(3), 485 490.

[6] Rada FH. (2018). Oxidative stress and some inflammatory biomarkers in patients with coronary heart disease. European journal of pharmaceutical and medical research, 5(1), 9 -12.

[7] Patrono C, Baigent C, Hirsh J and Roth G. (2008). Antiplatelet drugs: American College of Chest Physicians Evidence-Based Clinical Practice Guidelines (8th Edition). Chest,133, 199S-233S

[8] Cox D, Maree AO, Dooley M, Conroy R, Byrne MF and Fitzgerald DJ. (2006). Effect of enteric coating on antiplatelet activity of low-dose aspirin in healthy volunteers. Stroke, 37(8), 2153 - 2158.

[9] Patrono C, Ciabattoni G, Patrignani P, Pugliese F, Filabozzi P, Catella F, et al . (1985). Clinical pharmacology of platelet cyclooxygenase inhibition. Circulation, 72(6), 1177 - 1184.

[10] Lai KC, Lam SK, Chu KM, Wong BC, Hui WM, Hu WH, et al. (2002). Lansoprazole for the prevention of recurrences of ulcer complications from long-term low-dose aspirin use. New England Journal of Medicine, 346, $2033-8$.

[11] US Food and Drug Administration. (2010). Information for Healthcare Professionals: Concomitant Use of Ibuprofen and Aspirin. Rockville, MD: FDA Medwatch.

[12] Cattaneo M. (2007). Resistance to antiplatelet drugs: molecular mechanisms and laboratory detection. Journal of Thrombosis and Haemostasis, 5, 230-7.

[13] DiChiara J, Bliden KP, Tantry US, Hamed MS, Antonino MJ, Suarez TA, et al. (2007). The effect of aspirin dosing on platelet function in diabetic and nondiabetic patients: an analysis from the aspirin-induced platelet effect (ASPECT) study. Diabetes, 56, 3014-9.

[14] Savi P and Herbert JM. (2005).Clopidogrel and ticlopidine: P2Y12 adenosine diphosphate-receptor antagonists for the prevention of atherothrombosis. Seminars in Thrombosis and Hemostasis, 31(2), 174 - 183.

[15] Urban P, Macaya C, Rupprecht HJ, Kiemeneij F, Emanuelsson H, Fontanelli A, et al. (1998). Randomized evaluation of anticoagulation versus antiplatelet therapy after coronary stent implantation in high-risk patients: the multicenter aspirin and ticlopidine trial after intracoronary stenting (MATTIS). Circulation, 98, 2126 - 32.

[16] CAPRIE Steering Committee. (1996). A randomised, blinded, trial of clopidogrel versus aspirin in patients at risk of ischaemic events (CAPRIE). Lancet, 348, 1329-39.

[17] Rada FH. (2016). Clopidogrel and liver injury in diabetic patients. European journal of pharmaceutical and medical research, 3(6), 168-170.

[18] Hasan NA, Al Baghdadi MH and Rada FH. (2014). Assessment of adverse effect of atorvastatin with platelet P2Y12-ADP receptor antagonist on platelets aggregation and renal function in coronary heart disease treated patients. International journal of research in pharmacy and chemistry, 4(2), 274-282.

[19] Farid NA, Payne CD, Small DS, Winters KJ, Ernest CS II, Brandt JT, et al. (2007). Cytochrome P450 3A inhibition by ketoconazole affects prasugrel and clopidogrel pharmacokinetics and pharmacodynamics differently. Clinical Pharmacology and Therapeutics, 81, 735-41.

[20] Hochholzer W, Trenk D, Frundi D, Blanke P, Fischer B, Andris K, et al. (2005).Time dependence of platelet inhibition after a 600-mg loading dose of clopidogrel in a large, unselected cohort of candidates for percutaneous coronary intervention. Circulation, 111, 2560-4.

[21] Shuldiner AR,O'Connell JR, Bliden KP, Gandhi A, Ryan K, Horenstein RB, et al. (2009). Association of cytochrome P450 2C19 genotype with the antiplatelet effect and clinical efficacy of clopidogrel therapy. Journal of the American Medical Association, 302, 849-57. 
[22] Cuisset T , Frere C , Quilici J , Morange PE, Nait-Saidi L ,Carvajal J, et al .(2006). Benefit of a 600-mg loading dose of clopidogrel on platelet reactivity and clinical outcomes in patients with non-ST-segment elevation acute coronary syndrome undergoing coronary stenting.Journal of American College of Cardiology , 48 (7),1339 - 1345

[23] Serebruany VL, Malinin AI, Ferguson JJ, Vahabi J, Atar D and Hennekens CH. (2008). Bleeding risks of combination vs. single antiplatelet therapy: a meta-analysis of 18 randomized trials comprising 129,314 patients. Fundamental and Clinical Pharmacology, 22, 315-21.

[24] Rada FH, Hasan NA and Al Baghdadi MH. (2015). Hepatotoxicity of Combined Therapy of Atorvastatin with Platelet P2Y12-ADP Receptor Antagonist in Coronary Heart Disease Treated Patients. The Iraqi Postgraduate Medical Journal, 14(1), $108-113$.

[25] Rada FH. (2019).Peroxisome Proliferator- Activated Receptors Family Overview. European Journal of Pharmaceutical and Medical Research, 6(1), 167-170.

[26] Siller-Matula JM, Lang I, Christ G and Bernd Jilma B. (2008).Calcium-channel blockers reduce the antiplatelet effect of clopidogrel. Journal of the American College of, 52, 1557-1563.

[27] Bonello L, Tantry US, Marcucci R, Blindt R, Angiolillo DJ and Becker R. (2010).Working Group on High OnTreatment Platelet Reactivity. Consensus and future directions on the definition of high on-treatment platelet reactivity to adenosine diphosphate. Journal of American College of Cardiology, 56 (12), 919 - 933.

[28] Rehmel JL, Eckstein JA, Farid NA, Heim JB, Kasper SC, Kurihara A, et al. (2006). Interactions of two major metabolites of prasugrel, a thienopyridine antiplatelet agent, with the cytochromes P450. Drug Metabolism and Disposition, 34, 600-7.

[29] Braun 00, Johnell M, Varenhorst C, James S, Brandt JT, Jakubowski JA, et al.(2008). Greater reduction of platelet activation markers and platelet-monocyte aggregates by prasugrel compared to clopidogrel in stable coronary artery disease.Thrombosis and Haemostasis, 100, 626-33.

[30] Farid NA, Smith RL, Gillespie TA, Rash TJ, Blair PE and Kurihara A. (2007). The disposition of prasugrel, a novel thienopyridine, in humans. Drug Metabolism and Dispositions, 35 (7), 1096-8.

[31] Wiviott SD, Braunwald E, McCabe CH, Montalescot G, Ruzyllo W, Gottlieb S, et al. (2007). Prasugrel versus clopidogrel in patients with acute coronary syndromes. New England Journal of Medicine, 357, 2001-15.

[32] Small DS, Farid NA, Payne CD, Weerakkody GJ, Li YG, Brandt JT, et al. (2008).Effects of the proton pump inhibitor lansoprazole on the pharmacokinetics and pharmacodynamics of prasugrel and clopidogrel. Journal of Clinical Pharmacology, 48, 475-84.

[33] Erlinge D, Varenhorst C, Braun 00, James S, Winters KJ, Jakubowski JA, et al. (2008).Patients with poor responsiveness to thienopyridine treatment or with diabetes have lower levels of circulating active metabolite, but their platelets respond normally to active metabolite added ex vivo. Journal of American College of Cardiology, 52, 1968-77.

[34] Storey RF, Wilcox RG and Heptinstall S. (2002). Comparison of the pharmacodynamic effects of the platelet ADP receptor antagonists clopidogrel and AR-C69931MX in patients with ischaemic heart disease. Platelets, 13, 40713.

[35] Harrington RA, Stone GW, McNulty S, White HD, Lincoff AM, Gibson CM, et al. (2009). Platelet inhibition with cangrelor in patients undergoing PCI.New England Journal of Medicine, 361, 2318-29.

[36] Fugate SE and Cudd LA. (2006).Cangrelor for treatment of coronary thrombosis. Annals of Pharmacotherapy, 40, 925-30.

[37] Bhatt DL, Lincoff AM, Gibson CM, Stone GW, McNulty S, Montalescot G, et al. (2009). Intravenous platelet blockade with cangrelor during PCI. New England Journal of Medicine, 361, 2330 - 41.

[38] Steinhubl SR, OH JJ, Oestreich JH, Ferraris S, Charnigo R and Akers WS. (2008).Transitioning patients from cangrelor to clopidogrel: pharmacodynamic evidence of a competitive effect. Thrombosis Research, 121, 527-34.

[39] Rada FH. (2018).Antiplatelet Adequacy of Cytopentyl Triazolopyrimidine versus Clopidogrel in Patients with Coronary Heart Disease. Asian Journal of Pharmaceutical and Clinical Research, 11(12), 536 -539.

[40] Teng R and Butler K. (2010). Pharmacokinetics, pharmacodynamics, tolerability and safety of single ascending doses of ticagrelor, a reversibly binding oral P 2Y(12) receptor antagonist,in healthy subjects. European Journal of Clinical Pharmacology, 66, 487-96. 
[41] Wallentin L, Becker RC, Budaj A, Cannon CP, Emanuelsson H, Held C, et al. (2009). Ticagrelor versus clopidogrel in patients with acute coronary syndromes. New England Journal of Medicine, 361, 1045-57.

[42] Bhatt DL. (2007).Intensifying platelet inhibition-navigating between Scylla and Charybdis. New England Journal of Medicine, 357, $2078-81$.

[43] Born G and Patrono C. (2006). Antiplatelet drugs. British Journal of Pharmacology, 147 (1) , S241 - S251.

[44] Müller TH, Su CA, Weisenberger H, Brickl R, Nehmiz G and Eisert WG. (1990). Dipyridamole alone or combined with low-dose acetylsalicylic acid inhibits platelet aggregation in human whole blood ex vivo. British Journal of Clinical Pharmacology, 30(2), 179 - 186.

[45] Weinberger J. (2005).Adverse effects and drug interactions of antithrombotic agents used in prevention of ischaemic stroke. Drugs, 65, 461-71.

[46] Serebruany VL, Malinin AI, Eisert RM and Sane DC. (2004). Risk of bleeding complications with antiplatelet agents: metaanalysis of 338,191 patients enrolled in 50 randomized controlled trials. American Journal of Hematology, 75, 40-7.

[47] Thompson PD, Zimet R, Forbes WP and Zhang P. (2002). Meta-analysis of results from eight randomized, placebocontrolled trials on the effect of cilostazol on patients with intermittent claudication. American Journal of Cardiology, 90 (12), 1314 - 5.

[48] Biondi-Zoccai GG, Lotrionte M , Anselmino M , Moretti C, Agostoni P, Testa L,et al.(2008). Systematic review and meta-analysis of randomized clinical trials appraising the impact of cilostazol after percutaneous coronary intervention. American Heart Journal, 155 (6), 1081 - 8.

[49] Angiolillo DJ, Capranzano P, Goto S, Aslam M, Desai B, Charlton RK, et al.(2008). A randomized study assessing the impact of cilostazol on platelet function profiles in patients with diabetes mellitus and coronary artery disease on dual antiplatelet therapy: results of the OPTIMUS-2 study.European Heart Journal, 29, 2202 - 11.

[50] Hiratsuka M, Hinai Y, Sasaki T, Konno Y, Imagawa K, Ishikawa M, et al. (2007).Characterization of human cytochrome p450 enzymes involved in the metabolism of cilostazol. Drug Metabolism and Disposition, 35, 17302 .

[51] Gamssari F, Mahmood H, Ho JS, Villareal RP, Liu B, Rasekh A, et al.(2002). Rapid ventricular tachycardias associated with cilostazol use. Texas Heart Institute Journal, 29, 140-2.

[52] Suri A, Forbes WP, Bramer SL. (1999). Effects of CYP3A inhibition on the metabolism of cilostazol. Clinical Pharmacokinetics, 37, 61-8.

[53] Kereiakes DJ, Kleiman NS, Ambrose J, Cohen M, Rodriguez S, Palabrica T, et al.(1996). Randomized,double-blind, placebo-controlled dose-ranging study of tirofiban (MK-383) platelet IIb/IIIa blockade in high risk patients undergoing coronary angioplasty.Journal of American College of Cardiology,27 (3), 536 - 542 .

[54] Sharma HL and Sharma KK. (2007). Principles of pharmacology. 1st ed. Hyderabad: Paras medical publishers; Drugs affecting coagulation, fibrinolysis and platelet functions, 639, 703,704.

[55] Tcheng JE, Kereiakes DJ, Lincoff AM, George BS, Kleiman N, Sane DC, et al .(2001). Abciximab readministration: results of the ReoPro Readministration Registry. Circulation, 104 (8), 870 - 875.

[56] Gross PL and Weitz JI. (2009).New antithrombotic drugs. Clinical Pharmacology and Therapeutics, 86, 139-46.

\section{How to cite this article}

Latif SY, Issa MM, Hassan TN and Ali MH. (2020). Antiplatelet drugs overview. GSC Biological and Pharmaceutical Sciences, 10(1), 81-89. 